\title{
Azken hiru hamarkadetan Euskal Herriko kostaldean klima-aldaketak komunitate bentonikoetan (flora eta fauna) izan duen eragina
}

\author{
(The influence of Climate Change on benthic communities \\ (flora and fauna) of the Basque Coast during the last 3 decades)
}

\author{
Nahiara Muguerza*1, María Bustamante ${ }^{2}$, Isabel Díez ${ }^{1}$,Endika Quintano ${ }^{1}$, \\ Javier Tajadura $^{2}$, Iñaki Saiz-Salinas ${ }^{2}$, José María Gorostiaga ${ }^{1}$ \\ ${ }^{1}$ Botanika laborategia. Landare-Biologia eta Ekologia Saila. \\ Zientzia eta Teknologia Fakultatea (UPV/EHU) \\ ${ }^{2}$ Zoologia eta Animalia Zelulen Biologia Saila. \\ Zientzia eta Teknologia Fakultatea (UPV/EHU)
}

\begin{abstract}
LABURPENA: Errezela eratzen duten makroalgek, oro har, beherakada bortitza izan dute, eta horrek ekosistemako prozesuetan izango dituen eraginak ez dira oraindik ondo ezagutzen. Ikerketa honek Bizkaiko Golkoko hegoaldean 20 urte inguruko aldian substratu gogorreko ekosistema bentonikoak aztertu ditu, non Gelidium corneum makrofitoak beherakada nabarmena izan duen. Errezelak atzera egitearen edo aldaketaren tasa ikertu da, bai eta errezel-menpeko diren organismoen (algak eta ornogabeak) egitura taxonomiko eta funtzionalean ondorioak ere. Gure emaitzek erakutsi dute 2008an aldaketa garrantzitsuak zakarki gertatu zirela eta ondoren ez dela berreskuratze-seinalerik izan. Galera esanguratsuenak makroalga konplexu eta iraunkorrak diren formetan gertatu dira eta, aldiz, epifitoak diren eta ez diren suspentsiboroetan ere, alga sinple soropilduak ugaritu dira. Aldi berean, espezieen aberastasunak, algen dentsitateak eta flora eta faunaren ekitatibitateak gora egin dute. Ikerketaren 1. aldian (1998-2007), alga eta fauna espezie elkartuen talde koherenteak batera aldatzen dutela ikusten da. Hala ere, 2008tik aurrerantz finkatutako komunitate berrian algen eta ornogabeen arteko elkarte espezifikoak falta dira. Gure emaitzek flora eta fauna espezieen arteko ereduak daudela adierazten dute. Patroi hauek ekosistema bentonikoaren funtsezko espezie eta ezaugarri funtzionalen galeren ondorioak hobeto ulertzen lagunduko dute.
\end{abstract}

HITZ GAKOAK: Espezie elkartuak, Habitat biogenikoak, Makroalgen asanblajen beherakada, Ornogabeak.

\begin{abstract}
Canopy-forming macroalgae are globally facing drastic declines and the consequences for ecosystem processes remain still little known. The present investigation analyses in a 20 years period the retreat of shallow subtidal rocky benthos communities in the southern Bay of Biscay, where the macrophyte Gelidium corneum has suffered a notable decrease. The rate of change of canopy retreat was investigated as well as the effects of the decline on the taxonomic and functional structure of canopy-dependent organisms (algae and invertebrates). Our results show that major changes occurred abruptly in 2008, and no signs of recovery have been recorded afterwards. Major losses arose in complex perennial macroalgal forms together with epiphytic and no epiphytic suspensivores while simple turf-forming algae proliferated. At the same time, species richness, algal density and both flora and fauna evenness increased. There exist groups of coherent species of flora and fauna that covary during the first part of the studied period. However, from 2008 onwards the new established community lacked algal-invertebrate specific associations. Our results highlight patterns between flora and fauna species help to better understand the consequences of the loss of key species and functional traits for the benthic ecosystem.
\end{abstract}

KEYWORDS: Macroalgal assemblages decline, Associated species, Invertebrates, Biogenic habitats.

* Harremanetan jartzeko / Corresponding author: Nahiara Muguerza, Botanika laborategia. Landare-Biologia eta Ekologia Saila. Zientzia eta Teknologia Fakultatea (UPV/EHU). Sarriena auzoa z/g. 48940 Leioa. Bizkaia. Euskal Herria. - nahiara. muguerza@ehu.eus - https://orcid.org/0000-0002-4335-0663.

Nola aipatu / How to cite: Muguerza, Nahiara; Bustamante, María; Díez, Isabel; Quintano, Endika; Tajadura Javier; Saiz-Salinas, Iñaki; Gorostiaga, José María (2020). «Azkeneko hiru hamarkadetan Euskal Herriko kostaldean klima-aldaketak komunitate bentonikoetan (flora eta fauna) izan duen eraginaw; Ekaia, ale berezia 2020, 145-162. (https://doi.org/10.1387/ekaia.21006).

Jasoa: 11 uztaila, 2019; Onartua: 21 azaroa, 2019

ISSN 0214-9001 - elSSN 2444-3255 / (c) 2020 UPV/EHU

(i) (i) Obra hau Creative Commons Atribución 4.0 Internacional-en

lizentziapean dago 
Nahiara Muguerza, María Bustamante, Isabel Díez, Endika Quintano, Javier Tajadura, Iñaki Saiz-Salinas, José María Gorostiaga

\section{SARRERA}

Komunitate bentoniko naturaletan, errezela sortzen duten makroalgak oinarrizko ekoizle ekologikoak dira, eta funtsezko papera betetzen dute eremu epeletako substratu gogorreko itsas ekosistemetan. Azken hamarkadetan, mundu-mailan egin diren ikerketa askok erakutsi dute espezie egituratzaile hauek nabarmen gutxitu direla [1, 2, 3, 4]. Zenbait ebidentzia daude banaketa geografikoan aldaketak izan direla erakusten dutenak, bai eta alga oportunisten eta alga soropilduen eraginez makrofito hauek planeta osoko eskualdeetan habitata galdu dutela erakusten dutenak ere $[5,6,7,8]$. Ondorioz, komunitate bentonikoan aldaketa funtzional eta estrukturala gertatzen da $[9,10,11,12,13]$; izan ere, habitat biogeniko gisa oinarrizko baliabidea dira hainbat organismorentzat; hala nola, makroalgen mendeko diren ornogabeentzat, arrainentzat eta beste alga txikiagoentzat. Gainera, makrofito handiek harrapakariengandik eta beste zenbait faktoretatik babesten dituzte (estres abiotikoa), elikagaiak eman eta hiru dimentsioko espazioak eskaintzen dituzte, zeintzuetan taxon ezberdin asko ezartzen diren $[14,15,16]$. Beraz, espezie egituratzaile hauek ezinbestekoak dira sortzen dituzten komunitate konplexuetan, eta, gainera, kostaldeko ekosistemei funtsezko zerbitzuak ematen dizkiete. Autore askok frogatu dute kopa edo errezela galtzeak aldaketak eragiten dituela espezieen osaeran eta haitzetako ornogabe kopuruan, azken hauek gutxituz, eta horrek komunitate bentonikoak eskaintzen duen habitata sinplifikatzea dakarrela $[17,18,19,20,11,21,22,23]$. Aldaketa horiek guztiak klima-aldaketaren edota beste presio antropikoen ondorio direla uste da. Ikerketa gehienek ondorioztatzen dute kopa-estratua edo geruza galtzeko arrazoi nagusia eta, ondorioz, komunitate bentoniko osoaren aldaketa itsasoko tenperaturaren gorakada dela. Hala ere, berotze globalarekin batera, zenbait aldaketa gertatu dira beste ingurumen-aldagai batzuetan, hala nola, eguzki-erradiazioaren gorakada [24, 25], itsas dinamikaren alterazioak [26, 27], olatuen areagotzea [28] eta mantenugaien eskuragarritasunaren aldaketak [29], beste batzuen artean. Bestalde, tokiko eskalan, badakigu kostaldeko ekosistemak hainbat ingurumen- eta estres-faktoreren menpe daudela, kutsaduraren menpe, adibidez, eta horiek eragin handia izan dezaketela. Izan ere, komunitateen nolabaiteko aldakortasuna ingurumeneko estres-faktore antropogeniko edo naturalen arteko sinergiari egozten zaio [30]; adibidez, herbiboroak diren itsas trikuen presioari, zeinak makroalga egituratzaileak alga-soropilduez ordezkatzea lagundu edo azkartu dezakeen [31].

Horrela, egituratzaileak diren makroalgak ondo kudeatzen saiatzeko, funtsezkoa da hain konplexuak diren ekosistema horiek osatzen dituzten espezie ingeniarien galera-tasa identifikatzeko aldi baterako datu-serie handiak aztertzea. Gainera, galera horiek itsas ekosistemaren funtzionamenduan duten eragina hobeto ulertzeko, beharrezkoa da espezie bentonikoen konposizioan aldaketaren bat duten antzeko patroiei buruzko informazioa biltzea [32]. Ildo horretan, makroalgekin lotutako ornogabeak ingurumen- 
baldintzen adierazle onak dira, eta funtsezko zeregina dute ekosistemaren funtzionamenduan eta propietateetan [33]. Hala ere, seguruenik, mareaz azpiko komunitatearen laginak hartzeko zailtasunen ondorioz, gutxien aztertu den osagaietako bat da, itsas ekosistemetan garrantzi handia izan arren [34].

Tokian tokiko galerak zerk eragin dituen hobeto ulertzeko, Bizkaiko Golkoko hego-ekialdeko eremu batean eta hogei urteko tartean, espezieen aldaketak aztertu ditugu, bai eta sakonera gutxiko mareaz azpiko komunitate bentonikoen dibertsitate funtzionala ere (flora eta fauna). Aztertutako eremuan, Gelidium corneum espezie egituratzaileak mareaz azpiko zelai zabal eta konplexuak eratzen zituen 80ko eta 90eko hamarkadetan [35], baina azken urteotan nabarmen gutxitu da.

Gure ikerketaren arabera, aztertutako eremuan, flora- eta fauna-espezie koherenteak daude, eta ekosistema bentonikorako makrofitoen galeraren zergatiak hobeto ulertzen laguntzen duten patroiak erakusten dituzte.

\section{IKERKETA-EREMUA}

Aztertutako komunitateak Bizkaiko Golkoko hego-ekialdean daude, Euskal kostaldean $43^{\circ} 21^{\prime} 17.65^{\prime} \mathrm{N}-3^{\circ} 07^{\prime} 46.40^{\prime \prime} \mathrm{W}$ (1. irudia). Biogeografiari dagokionez, zona hori Atlantikoko ipar-ekialdeko eremu epel-beroan dago [36]. Kostalde zati hori Atlantiko ipar-ekialdeko olatuei zabalik dago eta itsas azaleko uraren bataz besteko tenperatura $12^{\circ} \mathrm{C}$ gradukoa da otsailean eta $22^{\circ} \mathrm{C}$-koa abuztuan [37], nahiz eta azken hamarkadetan gradu bat berotu den udan itsas azaleko ura, gradualki [38].

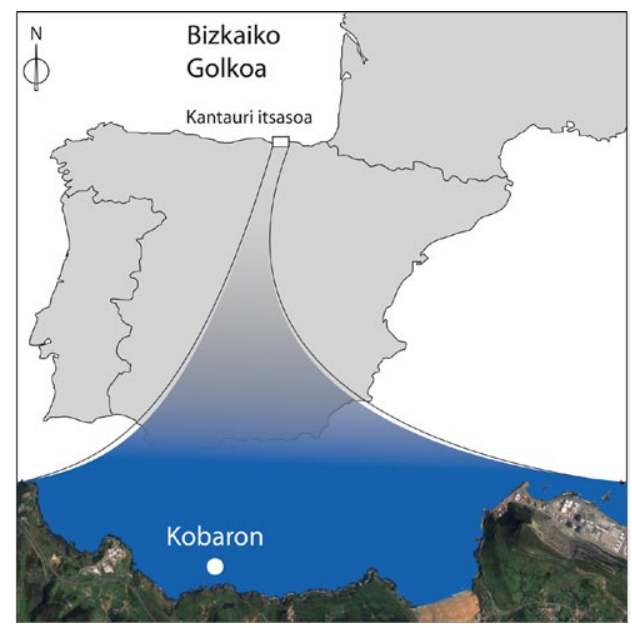

1.irudia. Ikerketa-eremua: Bizkaiko Golkoko hegoaldean laginketa-gunearen kokapena. 
Nahiara Muguerza, María Bustamante, Isabel Díez, Endika Quintano, Javier Tajadura, Iñaki Saiz-Salinas, José María Gorostiaga

\section{METODOLOGIA}

\subsection{Laginketa-metodologia}

Ikerketa honetan aztertutako datuak 1998. urtean hasitako Bentos taldearen jarraipen-lan baten parte dira. Bigarren laginketa bat egin zen 2000. urtean, eta ordutik 2017ra arte, urtero errepikatu da; beraz, lan honetan 19 laginketa-kanpainatako datu-serie luzea dago. Ikerketa-eremua erreferentziatzat hartu da: izan ere, potentzialki, ingurumen-baldintza onak ditu, kutsadura antropogenikoen nolabaiteko distantziara dagoelako.

2001etik 2017ra bitartean kostaldearekiko paralelo eta $5 \mathrm{~m}$-ko sakonerari jarraiki, transektu bat jarri zen (64 m-ko luzerakoa eta $4 \mathrm{~m}$-ko zabalerakoa). Horrela, 3-7 metroko sakonerako zatia estaliz, laginketa-eremu potentziala lortu zen. $2 \times 2$ m-ko 128 laginketa-azalera potentzialetatik zortzi aukeratu ziren, zoriz. Azalera bakoitzean, 4 koadrante $(50 \times 50 \mathrm{~cm})$ hautatu ziren zoriz. Horrela, urtean 32 koadrante $(\mathrm{n}=32)$ aztertu ziren. Hala eta guztiz ere, 1998an eta 2000n, bost metroko sakonerari jarraituz, 100 metro luzerako transektuan 10 metroan behin, koadrante bat jarri zen $(n=10)$. Guztira, ikerketa honetan 564 laginketa-unitate aztertu dira.

Koadrante bakoitzean, begiz, landarediaren eta makroornogabeen estaldura-estimazioak egin ziren. Brawn-Blanquetek (1951) proposatutako estaldura-eskala erabiliz kalkulatu zen ugaritasuna: +: presentzia; 1: 1-5\%; 2: $5-25 \% ; 3: 25-50 \%$; $4: 50-75 \%$; $5: 75-100 \%$.

\subsection{Erantzun-aldagaiak eta analisi estatistikoa}

Ikerketa honetan, ezaugarri morfo-funtzionalak nahiz taxonomikoak erabili dira. Aldagai funtzionalak kalkulatzeko, lagin bakoitzeko taxonen estaldurak 3 ezaugarritan aztertu ziren ornogabeen kasuan (bizi-formak, helduen mugikortasuna eta talde trofikoak), eta algen kasuan, beste 3 ezaugarri desberdinetan (bizi-formak, morfologia eta banaketa bertikala). Ornogabeen ezaugarri edo bereizgarri biologikoek haien jokaera islatzen dute behar duten habitatean, bai eta helduen mugikortasuna eta haien estrategia trofikoa ere. Algen ezaugarriek bizi-forma, konplexutasun morfologikoa eta banaketa bertikal estratifikatua (geruzak) adierazten dituzte. Azterturiko aldagai taxonomikoak honako hauek dira: aberastasuna, estaldura, dentsitatea eta ekitatibitatea, eta banaka eta bereizita kalkulatu ziren ornogabeetan eta algetan. Patroiak edo joerak bilatzeko asmoz, aldagaiak urte bakoitzeko edo laginketa bakoitzeko bataz bestekoaren arabera irudikatu dira.

Komunitatearen egitura taxonomikoaren espazio- eta denbora-aldagarritasuna testuinguru anitz batean aztertu zen. Bi ikuspegi erabili ziren: $Q$-mode analisia, laginen artean patroiak detektatzeko (hau da, urteen artean) [39] eta $R$-mode analisia aldagaien, hau da, ornogabeen eta algen ar- 
teko harremanak aztertzeko [40]. Horretarako, Bray-Curtis indizearen bidez, lagin bikoteen arteko antzekotasuna zehaztu zen. Datu multibarianteak irudikatzeko CLUSTER eta Non-metric multidimensional scaling ( $n$-MDS) sailkapen eta ordenazio-diagramak egin ziren. SIMPER analisiak erabiliz jakin zen zein ornogabe- edota alga-taxon ziren behatutako patroiak gehien esplikatzen zituztenak.

Laginen artean modu eta era berean aldatzen diren taxonak bereizteko, Coherent Plots-ak egin ziren, Shade Plots-ekin konbinatuta eta AsoziazioIndizea erabilita [41]. Soilik 50 taxon ugarienak sartu ziren analisian, eta kasu gehienetan, azterturiko urteetan, gutxienez \% 1eko estaldura izan zuten. Egindako analisi estatistiko guztiak eraldatu gabeko datuekin egin dira, eta PRIMER 7 pakete informatikoa erabiliz (Plymouth Routines in Multivariate Ecological Research software programme) [42].

\section{EMAITZAK ETA EZTABAIDA}

Komunitateak elkarrengandik ondo bereizitako bi taldetan banatzen direla erakusten dute CLUSTER zein nMDS analisiek (2. irudia). CLUSTER sailkapen-analisian 2 taldeak \% 32ko antzekotasun-portzentaje bat dutela ikusten da. $n M D S$ ordenazio grafikoan azaltzen den bezala, 1. taldearen inbentarioak 1998tik 2007ra arteko laginketa-kanpaina guztiei dagozkienak dira (lehen aldia); 2. taldean, berriz, 2008tik 2017ra arteko inbentario guztiak sartzen dira (bigarren aldia). Gainera, 1. aldiko urteak diagraman beren artean gertuago kokatuta daudenez, nabarmen erakusten dute beren artean parekoagoak direla; kontran, 2. aldiko urteak gehiago bereizten dira. Beraz, nabarmena gertatzen da Euskal kostaldeko komunitate bentonikoek aldaketa esanguratsu eta bortitza izan dutela beren konposizioan, bereziki 2008tik aurrera. Errezela sortzen duten makrofitoak, haiekin batera doazen flora-espezieak eta horiei lotutako ornogabeak ia desagertu egin dira, eta lehenengo aldian (1998-2007) agertzen ez ziren alga-espeziez osatutako errezel-geruzarik gabeko komunitate berri bati egiten diote bidea. Bigarren aldian (2008-2017), ez dago flora- eta fauna-espezieen elkarterik.

SIMPER analisiaren emaitzek komunitate bentonikoetan 1. aldian (1998-2007) honako taxon hauek (alga-espezieak eta ornogabeak) nagusi zirela adierazi zuten: Gelidium corneum, Mesophyllum lichenoides, Plocamium cartilagineum, Dictyota dichotoma, Electra pilosa, Obelia geniculata, Rocellaria dubia, Crisia spp. eta Rhodymenia pseudopalmata (1. taulan). Aitzitik, 2008tik 2017ra bitartean (2. aldia), joera kontrakoa da: taxon horiek murriztu egin ziren, nahiz eta honako alga-espezie hauek nabarmen hazi ziren: Aphanocladia stichidiosa, Zanardinia typus, Codium decorticatum, Aglaothamnion byssoides, Falkenbergia rufolanosa, Heterosiphonia japonica eta Asparagopsis armata. 
Nahiara Muguerza, María Bustamante, Isabel Diez, Endika Quintano, Javier Tajadura, Iñaki Saiz-Salinas, José María Gorostiaga
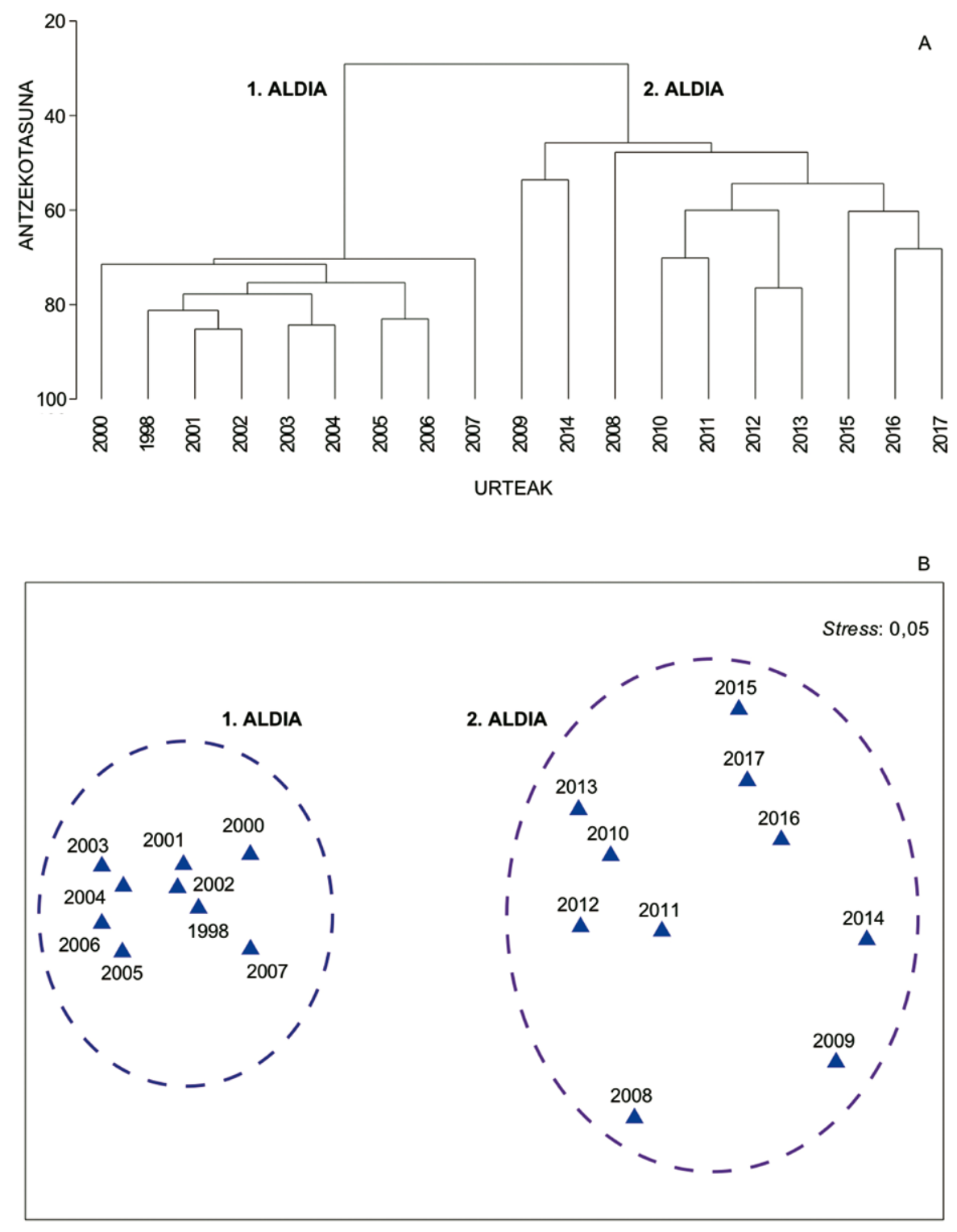

2. irudia. Taxonen konposizioa eta ugaritasunaren Cluster sailkapen-analisia (A) eta nMDS ordenazio-diagrama (B) Bray-Curtis-en antzekotasun-indizean oinarritua. 
1. taula. SIMPER analisian esanguratsuak diren espezieak (*:fauna). 1. aldia: 1998-2007. 2. aldia: 2008-2017.

\begin{tabular}{lcc}
\hline \multicolumn{1}{c}{ Espezieak } & \multicolumn{2}{c}{ BATAZ BESTEKO UGARITASUNA } \\
1. Aldian & 2. Aldian \\
\hline Gelidium corneum & $\mathbf{7 8 , 3}$ & 6,9 \\
Mesophyllum lichenoides & $\mathbf{7 1 , 4}$ & 36,5 \\
Dictyota dichotoma & $\mathbf{2 1 , 9}$ & 0,1 \\
Plocamium cartilagineum & $\mathbf{2 0 , 5}$ & 1,9 \\
Electra pilosa* & $\mathbf{1 5 , 0}$ & 0,2 \\
Aphanocladia stichidiosa & - & $\mathbf{1 2 , 5}$ \\
Codium decorticatum & 0,9 & $\mathbf{1 2 , 5}$ \\
Zanardinia typus & 3,8 & $\mathbf{1 5 , 2}$ \\
Aglaothamion tenuissimum & 1,1 & 11,0 \\
Rocellaria dubia* & $\mathbf{1 5 , 1}$ & $\mathbf{7 , 7}$ \\
Falkenbergia rufolanosa & 1,8 & $\mathbf{7 , 0}$ \\
Crisia spp.* & $\mathbf{5 , 7}$ & 0,2 \\
Asparagopsis armata & 0,3 & $\mathbf{5 , 6}$ \\
Rhodymenia pseudopalmata & $\mathbf{6 , 0}$ & 0,6 \\
\hline
\end{tabular}

1. aldia, 1998tik 2007. urtera artekoa, 3. irudiko grafikoan ikusten da alga eta ornogabeen espezie elkartuen talde koherenteek osatzen dutela (I-VI taldeak). Eta bigarren aldia, berriz (VII-XV taldeak), bereziki 2008. urtean agertu eta ordutik nagusi diren alga espezieek ia bakarrik karakterizatzen dute.

Lehen aldiaren ezaugarri diren espezie elkartuetan, Cystoseira baccata eta Gelidium corneum makrofito handiak, bai eta beste honako hauek ere: Dictyota dichotoma eta Plocamiun cartilagineum espezie epifitoak, oin-espezieak (hala nola, Cryptopleura ramosa, Acrosorium ciliolatum eta Rhodymenia pseudopalmata) eta Mesophyllum lichenoides espezie inkrustatzaileak ageri dira. Algen artean hazten diren animalien artean, briozooak diren Electra pilosa eta Crisia spp., Conopeum seurati eta Turbicellepora magnicostata ornogabeak eta horiekin batera substratu gogorrean hazten diren beste zenbait espezie ere azaltzen dira; hauek, esaterako: hidrozooak diren Rocellaria dubia, Laomedea exigua eta Obelia geniculata. ShadePlot grafikoak erakutsi duenez, espezie horietako gehienak bigarren aldian ez dira horren ugariak edo desagertu egin dira. 2008tik aurrera, sortzen diren taldeak hainbat alga soropilduz osatuta daude; honako hauetaz, esaterako: Aphanocladia stichidiosa, Aglaothamnion spp. eta Heterosiphonia japonica. Bestalde, Codium decorticatum makrofitoa eta Zanardinia typus inkrustanteaz osaturiko taldeak ere azaltzen dira. 
Nahiara Muguerza, María Bustamante, Isabel Díez, Endika Quintano, Javier Tajadura, Iñaki Saiz-Salinas, José María Gorostiaga

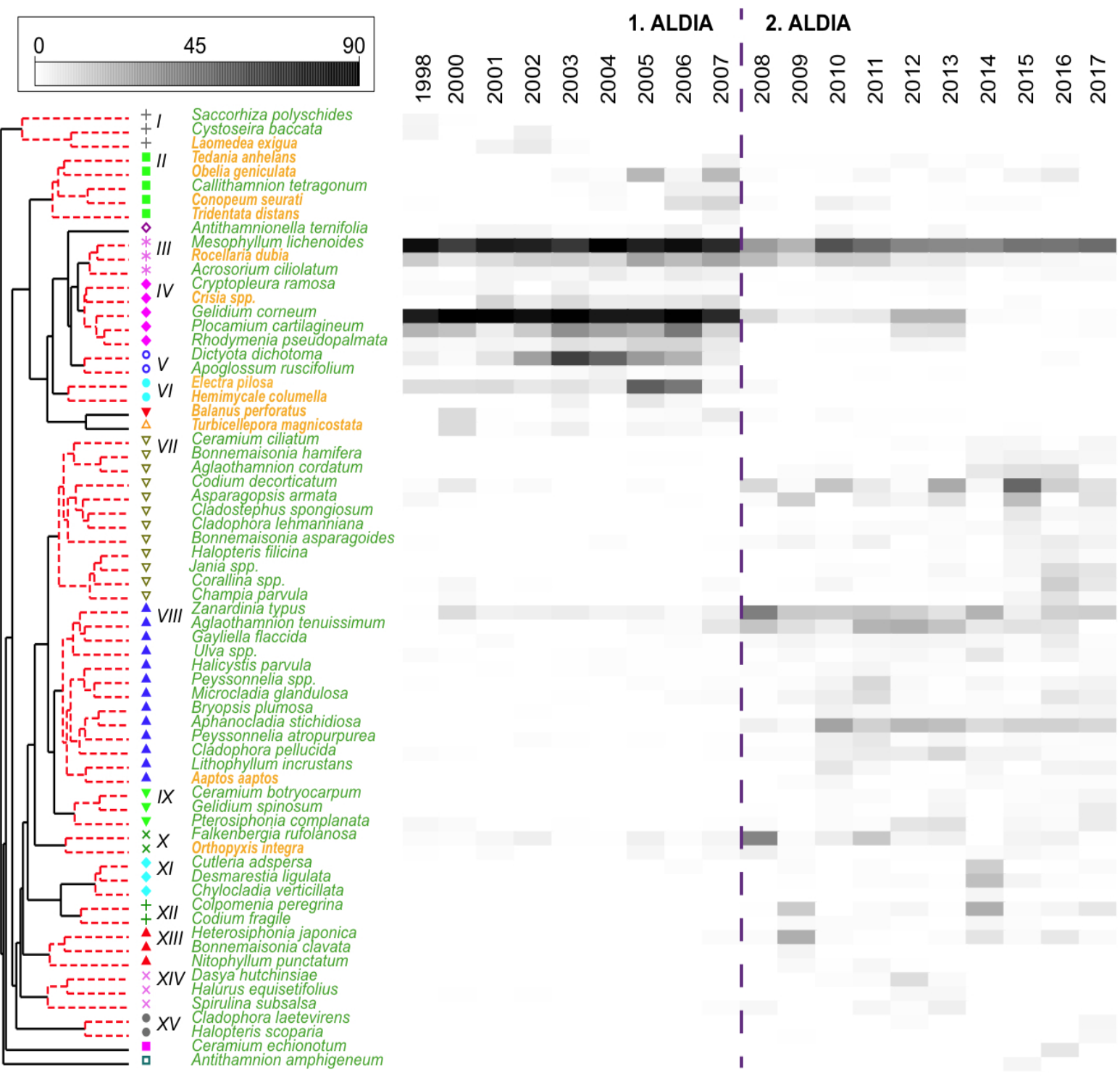

3. irudia. Taxonen arteko Asoziazio-Indizean oinarritutako datu-matrizearen Shade-plot-a, non 15 talde koherente erakusten diren (I-XV) aztertutako 19 urteetarako. Taxon berdeak alga-espezieak dira eta laranjak ornogabeak. Marra eten moreak bi aldiak bereizten ditu sailkapen- eta ordenazio-analisietan erakusten den moduan. Espazio hutsek urte jakin baterako taxon baten falta adierazten dute. Marra beltz etengabeak aldagai-multzo esanguratsuak $(\mathrm{p}>0,05)$ adierazten ditu eta marra eten gorriak zein taxon taldetan hipotesi nulua ezin den baztertu $(p>0,05)$ adierazten du. 
Aztertutako aldagai guztien artean, aldaketa handienak algen eta ornogabeen estalduran gertatu dira. Hortaz, gure emaitzak bat datoz mundumailan errezel estratuaren gainbehera erakusten duten beste lan batzuetan ikusitakoarekin $[43,11]$. Ikerketa hasi genuenetik, bentosaren estaldurak behera egin du, 4.A irudian ikusten den bezala, eta, gainera, puntualki gainbehera handiak izan ditu. Espezieen aberastasunari dagokionez (4.B irudia), bentosa osatzen duten ornogabe eta alga espezieak ugaritu egin dira ikerketak iraun duen denboran. Nolanahi ere, aztertutako aldian zenbait murrizpen ere antzeman dira, baina denboran ez dira beti berdinak izan algetan eta ornogabeetan.

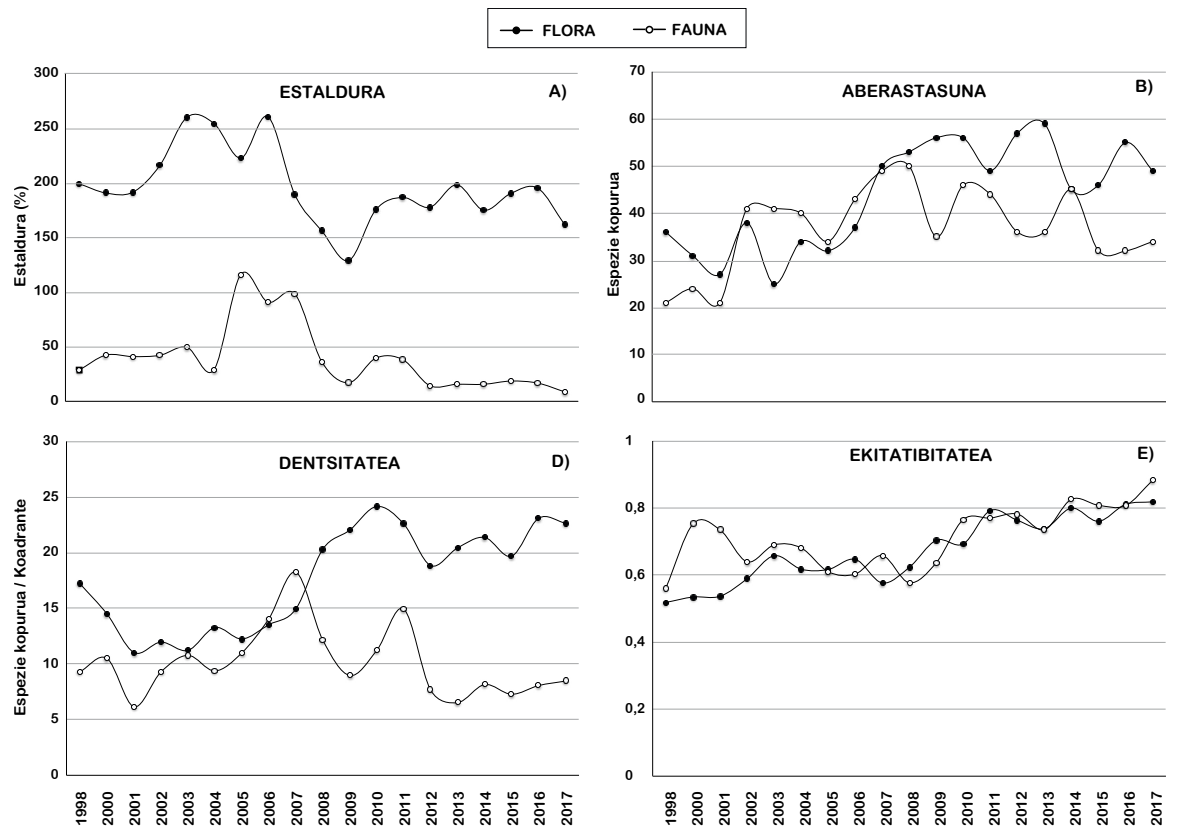

4. irudia. Alga eta ornogabeen Estaldura (A), Aberastasuna (B), Dentsitatea (D) eta Ekitatibitatearen (E) denbora-aldagarritasuna. Desbideratze-barrak kendu egin dira grafikoaren irakurketa errazteko.

Lehen aldiko dentsitate-balioei dagokienez (4.D irudia), algen eta ornogabeen dentsitatea nahiko antzekoa izan zen, baina bigarren aldian algen dentsitatea nabarmen handiagoa izan zen. 2008az geroztik, algen formen dentsitateak gora egin du, eta ornogabeenak, behera. Azkenik, aztertutako komunitateen ekitatibitatea (4.E irudia) nahiko egonkorra izan da aztertutako lehen aldian (1998-2007). Hala ere, 2008az geroztik, bai flora eta bai fauna nabarmen ugaritu dira. 
Nahiara Muguerza, María Bustamante, Isabel Díez, Endika Quintano, Javier Tajadura, Iñaki Saiz-Salinas, José María Gorostiaga
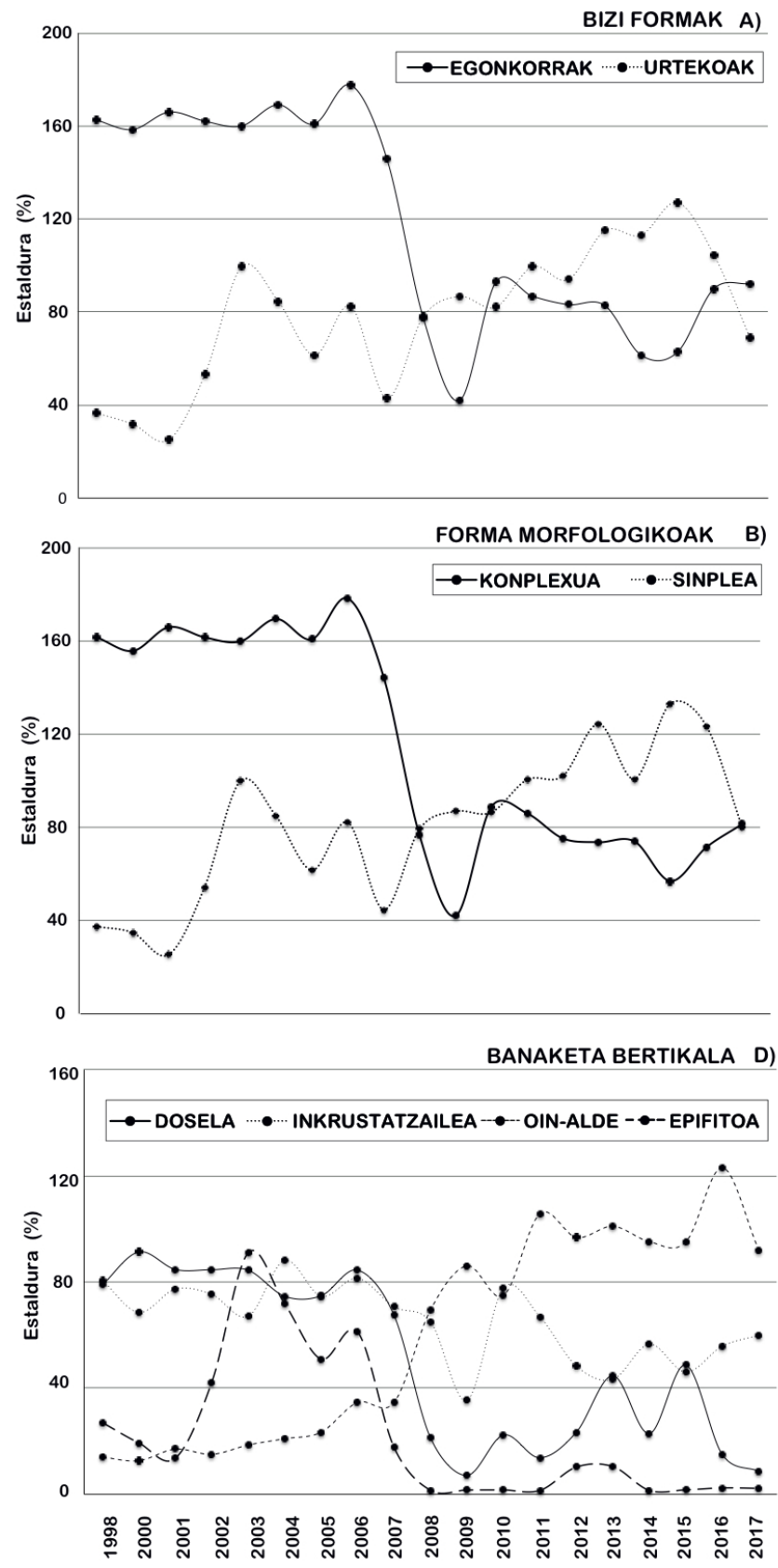

5. irudia. Alga komunitateen ezaugarri morfo-funtzionalen denbora-aldagarritasuna: Bizi-formak (A), Morfologia (B) eta Banaketa bertikaleko geruzak (D). Desbideratze-barrak kendu egin dira grafikoaren irakurketa errazteko. 
Errezela galtzearen ondorioz, komunitateetako funtsezko parametroetan aldaketa handiak izan dira. Floraren nahiz faunaren estalduran izan den gutxitze hori emaitza adierazgarria da, ekosistemaren prozesu garrantzitsuetan izaten duelako eragina, biomasaren ekoizpenean, adibidez [46, 43]. Komunitate berri honetan ez dago espezie egituratzaile nagusirik, eta kolonizatzeko dagoen espazio berriak algen eta ornogabeen aberastasuna handitzen laguntzen du, ingurumen estresatuetan gertatzen den bezala [47]. Gainera, ekitatibitatea handitu egiten da bigarren aldian, eta horrek adierazten du ez dela gainerakoen gaineko abantailarik hartu, baizik eta alderantziz, espazioaren banaketa ekitatiboagoa dela.

Ikuspegi funtzionala kontuan hartuta, komunitateek patroi ezberdinei jarraitzen diete, bi aldiotan. Algen banaketa bertikaleko geruzei dagokienez (5. irudia), lehenengo aldian errezela sortzen duten espezieak eta espezie inkrustatzaileak oin-espezieak baino ugariagoak dira; egonkor mantentzen dira, aldaketarik gabe; aldiz, geruza epifitoan aldaketak gertatu dira. 2008an, errezel-geruza nabarmen txikitu zen, eta, aldiz, estratu inkrustatzailea, gutxi. Gainera, oin-espezieentzat oso bestelako patroia ikusi zen, 2007 tik ikerketa amaitu artean hazkunde handia izan zutela ikusi baita. Bigarren aldian (2008-2017), oin-geruza nagusitu zen, errezelen kaltetan, eta gainera, azken hauek fluktuazio moderatuak izan dituzte. Geruza inkrustatzailea murriztu egin da, baina, hala ere, kopuru nahikoa handitan ageri da tarte horretan; geruza epifitikoa, aldiz, ia desagertu egin da.

Makroalga-talde funtzionalei dagokienez (5.B eta 5.D), aldaketa garrantzitsuenak morfologian (forma sinpleak edo konplexuak) eta bizi-formetan (urtekoak edo iraunkorrak) antzematen dira. 1998tik 2006ra, espezie konplexuak nagusi dira, eta komunitatean egonkor mantentzen dira. 2007 eta 2009 urteen artean, espezie konplexuetan beherakada nabarmena nabari da, geroago apalduko dena, eta, aldi berean, urteko formak gehitu egiten dira ikerketa amaitu arteko denboran. Bizi-formen azterketak forma morfologikoen antzeko patroia erakutsi du. Espezie iraunkorrak nabarmen ugariagoak dira eta egonkor mantentzen dira 1998tik 2006ra bitartean. 2006tik 2009ra bitartean, aldiz, nabarmena izan da beherakada. 2008. urtetik aurrera, urteko formak gehitu egiten dira. Bigarren aldian, urteko espezieen eta espezie iraunkorren ugaritasun-balioak antzekoak dira.

Ornogabeei dagokienez (6. irudia), 2008ra arte, forma epifitoek eta epibentonikoek antzeko ugaritasun-balioak erakutsi zituzten. Espezie epifitoak 1998. urtean bakarrik ageri dira kopuru txikiagoetan. 2006an nabarmen ugaritu ziren, baina gero izugarri murriztu ziren, eta konparaziora, forma epibentonikoak nabarmen ugaritu ziren. Ornogabe epibentonikoak epifitoak baino gehiago ziren arren, hauek ere murriztuz joan ziren ikerketa bukatu bitartean. Ornogabe helduen mugitzeko gaitasunari dagokionez, forma sesilak bakarrik aldatu ziren aztertutako aldian, eta horren ugariak ez diren mugimendu-ahalmena duten ornogabeak egonkor mantendu ziren. Beraz, 2006. urtean hazkunde nabarmena izan zen, eta gero, azterketa amaitu artekoan, gainbehera handia. 
Nahiara Muguerza, María Bustamante, Isabel Díez, Endika Quintano, Javier Tajadura, Iñaki Saiz-Salinas, José María Gorostiaga
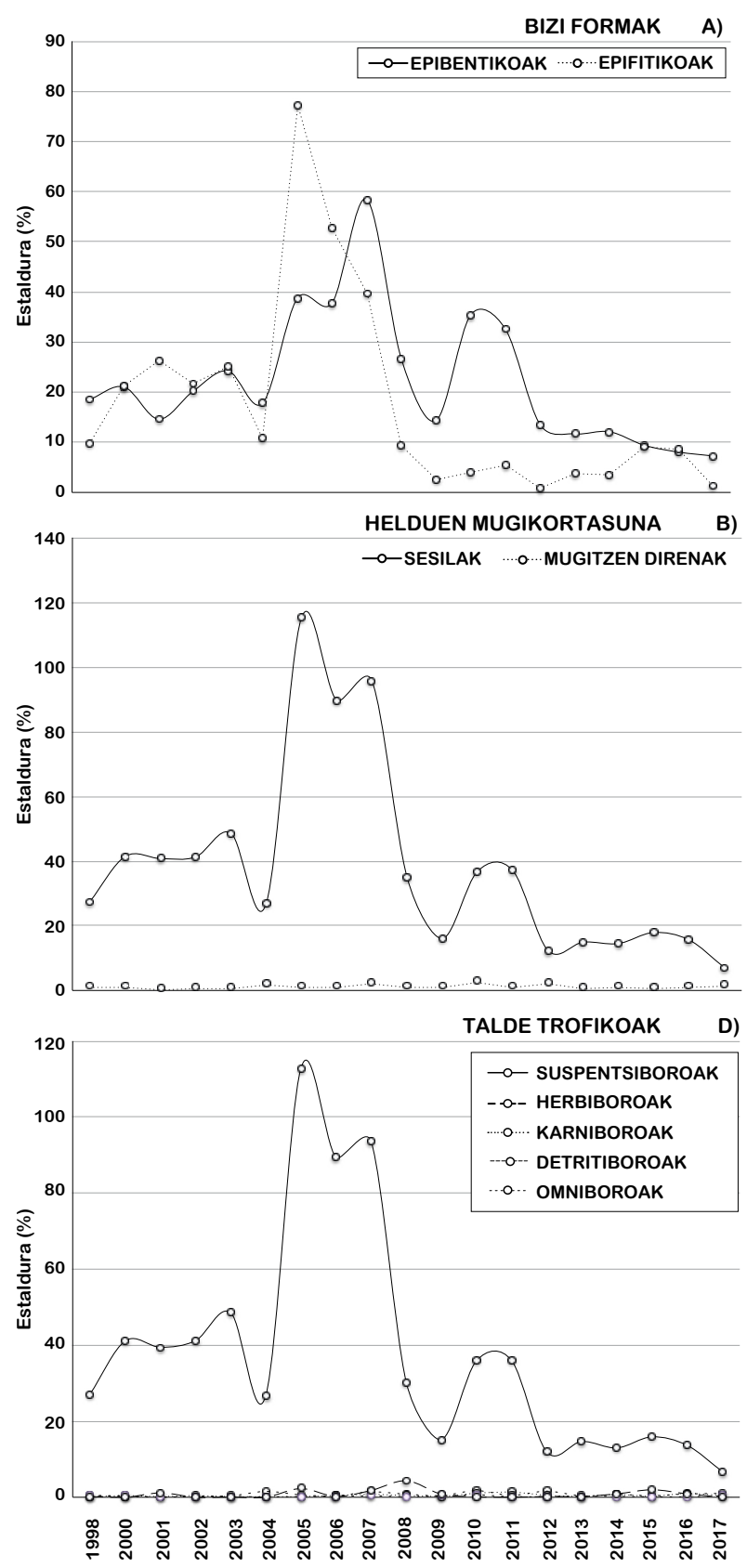

6. irudia. Ornogabe komunitateen ezaugarri funtzionalen denbora-aldagarritasuna: Bizi-formak (A), Helduen mugikortasuna (B) eta Talde trofikoak (D). Desbideratze-barrak kendu egin dira grafikoaren irakurketa errazteko. 
Gorago deskribatutako ornogabe helduen mugikortasunaren bilakaerak, talde trofikoetan behatutakoaren antzeko portaera erakutsi du, haietan suspentsiboroek heldu sesilek dituzten bariazio berberak erakutsi baitituzte. Suspentsiboroak dira ikerketan zehar ugariena den estrategia trofikoa, eta aldaketa nabarmenak erakutsi dituen bakarra da; beste estrategia trofiko batzuk, aldiz, egonkor iraun dute denboran.

Oro har, komunitateak maila funtzionalean aldaketa nabarmenak izaten ditu. Errezel-geruza galtzeak eta estratu basala ugaritzeak espezie iraunkor eta konplexuen gainbehera dakarte, eta urteroko forma sinpleen ugaritzearen ondorio dira. Makrofito handirik ez dagoenez, fauna epifitoaren nahiz basalaren ugaritasuna apaldu egiten da. Floraren aldaketak eragin handiagoa du ornogabe sesiletan eta estrategia trofiko suspentsiboroan. Fauna suspentsiboroa galtzearen ondorioz, sistema pelagiko eta bentonikoen arteko energia-lotura larri alda daiteke. Emaitza hauek nabarmentzen dute komunitatea ez bakarrik ikuspegi taxonomikotik baizik eta funtzionaletik ere aztertzea oso garrantzitsua dela. Izan ere, oso baliagarria da ekosisteman gertatzen diren aldaketa estrukturalak eta funtzionalak antzemateko [48, 49]. Gainera, gure ikerketak erakusten du aldaketa azkar bat gertatu dela. Ildo horretan, argi dago egoera alternatiboak bat-batean gertatu ahal direla ingurumen-aldagaietan muturreko aldaketak gertatzen direnean [44, 45, 43].

\section{ONDORIOAK}

Ikerketa hau tokikoa den arren, aztertutako denbora-tartea zabala da, eta hori erreferentziazkoa gerta daiteke komunitate heldu baten gainbehera ikusteko, bai eta berria nola ezartzen den ikusteko ere. Aztertutako azken urtean (2017), beste makrofitoek ez dute Gelidium corneum espezie egituratzailea ordezkatu. Komunitate garatu batek beharrezkoa duen substratu biogenikoa sortzen duen ordezkorik ez dago dagoeneko. Beraz, beharrezkoa da $G$. corneum itsas hondoko belardiak nagusi diren stocken jarraipena egitea eta haiek zaintzea euskal kostaldean, horiek dibertsitatearen gordailu izan daitezkeelako. Gainera, prozesu bentonikoen eta pelagikoen arteko loturak eta haien efektua ekosistemaren funtzionamenduan hobeto ulertzeak etorkizuneko kudeaketa-estrategien proiekzioa sostengatuko luke.

\section{BIBLIOGRAFIA}

[1] HARLEY, C.D.G., ANDERSON, K.M., , K.W., , J.P., , R.L., T.A. eta, M.H. 2012. «Effects of climate change on global seaweed communities». Journal of Phycology, 48, 1064-1078. 
Nahiara Muguerza, María Bustamante, Isabel Díez, Endika Quintano, Javier Tajadura, Iñaki Saiz-Salinas, José María Gorostiaga

[2] WERNBERG, T., BENNETT, S., BABCOCK, R.C., BETTIGNIES, T., CURE, K., DEPCZYNSKY, M., DUFOIS, F., FROMONT, J., FULTON, C.J., HOVEY, R.K., HARVEY, E.U., HOLMES, T.H., KENDRICK, G.A., RADFORD, B., SANTANA-GARCON, J., SAUNDERS, B.J., SMALE D.A., THOMSEN, M.S., TUCKETT C.A., TUYA, F., VANDERKLIFT, M.A. eta WILSON, S. 2016. "Climate-driven regime shift of a temperate marine ecosystem». Science, 353, 169-172.

[3] MARTÍNEZ, B., RADFORD, B., THOMSEN, M.S., CONNELL, S.D., CARREÑO, F., BRADSHAW, C.J.A., FORDHAM, D.A., RUSSELL, B.D., GURGEL, C.F.D. eta WERNBERG, T. 2018. «Distribution models predict large contractions of habitat-forming seaweeds in response to ocean warming». Diversity and Distributions, 24, 1350-1366.

[4] BORJA, A., CHUST, G., FONTÁN, A., GARMENDIA, J.M. eta UYARRA, M.C. 2018. «Long-term decline of the canopy-forming algae Gelidium corneum, associated to extreme wave events and reduced sunlight hours, in the southeastern Bay of Biscay». Estuarine, Coastal and Shelf Science, 205, $152-160$.

[5] MIESZKOWSKA, N., KENDALL, M.A., HAWKINS, S.J., LEAPER, R., WILLIAMSON, P., HARDMAN-MOUNTFORD, A. eta SOUTHWARD. A.J. 2006. "Changes in the range of some common rocky shore species In Britain - A Response To Climate Change?». Marine Biodiversity, 138, 241-251.

[6] MÜLLER, R., LAEPPLE, T., BARTSCH, I. eta WIENCKE, C. 2009. «Impact of oceanic warming on the distribution of seaweeds in polar and coldtemperate waters». Botanica Marina, 52, 617-638.

[7] AIROLDI, L. eta BECK, M.W. 2007. «Loss, status and trends for coastal marine habitats of Europe». Oceanography and Marine Biology, 45, 345405.

[8] TAKOLANDER, A., CABEZA, M. eta LESKINEN, E. 2017. «Climate change can cause complex responses in Baltic Sea macroalgae: A systematic review». Journal of Sea Research, 123, 16-29.

[9] DÍEZ, I., SANTOLARIA, A., MUGUERZA, N. eta GOROSTIAGA, J.M. 2013. «Measuring restoration in intertidal macrophyte assemblages following sewage treatment upgrade». Marine Environmental Research, 84, 31-42.

[10] BENEDETTI-CECCHI, L., PANNACCIULli, P., BULLERI, F., MOSCHELLA, P.S., AIROLDI, L., RELINI, G. eta CINELLI, F. 2001. «Predicting the consequences of anthropogenic disturbance: largescale effects of loss of canopy algae on rocky shores». Marine Ecology Progress Series, 214, 137-150.

[11] WERNBERG, T., THOMSEN, M.S., TUYA, F. eta KENDRICK, G.A. 2011. «Biogenic habitat structure of seaweeds change along a latitudinal gradient in ocean temperature». Journal of Experimental Marine Biology and Ecology, 400, 264-271. 
[12] HAWKINS, S.J., SUGDEN, H.E., MIESZKOWSKA, N., MOORE, P.J., POLOCZANSKA, E., LEAPER, R., HERBERT, R.J.H., GENNER, M.J., MOSCHELLA, P.S., THOMPSON, R.C., JENKINS, S.R., SOUTHWARD, A.J. eta BURROWS, M.T. 2009. «Consequences of climate-driven biodiversity changes for ecosystem functioning of North European rocky shores». Marine Ecology Progress Series, 396, 245-259.

[13] MUGUERZA, N., DÍEZ, I., QUINTANO, E., BUSTAMANTE, M. eta GOROSTIAGA, J.M. 2017. «Structural impoverishment of the subtidal vegetation of southeastern Bay of Biscay from 1991 to 2013 in the context of climate change». Journal of Sea Research, 130, 166-179.

[14] BERTOCCI, I., ARENAS, F., MATIAS, M., VASELLI, S., ARAÚJO, R., ABREU, H., PEREIRA, R., VIEIRA, R. eta SOUSA-PINTO, I. 2010. «Canopy-forming species mediate the effects of disturbance on macroalgal assemblages on Portuguese rocky shores». Marine Ecology Progress Series, 414, 107-116.

[15] BULLERI, F., BENEDETTI-CECCHI, L., CUSSON, M., MAGGI, E., ARENAS, F., ASPDEN, R., BERTOCCI, I., CROWE, T.P., DAVOULT, D., ERIKSSON, B.K.S., FRASCHETTI, S., GOLLÉTY, C., GRIFFIN, J.N., JENKINS, S.R., KOTTA, J., KRAUFVELIN, P., MOLIS, M., SOUSA PINTO, I., TERLIZZI, A., VALDIVIA, N. eta PATERSON, D.M., 2012. «Temporal stability of European rocky shore assemblages: variation across a latitudinal gradient and the role of habitat-formers». Oikos, 121, 1801-1809.

[16] STENECK, R.S., GRAHAM, M.H., BOURQUE, B.J., CORBETT, D., ERLANDSON, J.M., ESTES, J.A. eta TEGNER, M.J. 2002. «Kelp forest ecosystem: biodiversity, stability, resilience and future». Environmental Conservation, 29, 436-459.

[17] LILLEY, S.A. eta SCHIELD, D.R. 2006. «Community effects following the deletion of a habitat-forming alga from rocky marine shores». Oecologia, 148, 672-681.

[18] WIKSTRÖM, S.A. eta KAUTSKY, L. 2007. «Structure and diversity of invertebrate communities in the presence and absence of canopy-forming $\mathrm{Fu}$ cus vesiculosus in the Baltic Sea». Estuarine Coastal and Shelf Science, 72, 168-176.

[19] NORDERHAUG, K.M., CHRISTIE, H. eta FREDRIKSEN, S. 2007. «Is habitat size an important factor for faunal abundances on kelp (Laminaria hyperborea)?». Journal of Sea Research, 58, 120-124.

[20] TUYA, F., LARSEN, K. eta PLATT, V. 2011. «Patterns of abundance and assemblage structure of epifauna inhabiting two morphologically different kelp holdfasts». Hydrobiologia, 658, 373-382.

[21] BUSTAMANTE, M., TAJADURA, J., DÍEZ, I. eta SAIZ-SALINAS, J.I. 2017. «The potential role of habitat-forming seaweeds in modeling benthic ecosystem properties». Journal of Sea Research, 130, 123-133. 
Nahiara Muguerza, María Bustamante, Isabel Díez, Endika Quintano, Javier Tajadura, Iñaki Saiz-Salinas, José María Gorostiaga

[22] FILBEE-DEXTER, K. eta SCHEIBLING, R.E. 2014. «Sea urchin barrens as alternative stable states of collapsed kelp ecosystems». Marine Ecology Progress Series, 495, 1-25.

[23] FRANCO, J.N., WERNBERG, T., BERTOCCI, I., DUARTE, P., JACINTO, D., VASCO-RODRIGUES, N. eta TUYA, F. 2015. «Herbivory drives kelp recruits into 'hiding' in a warm ocean climate». Marine Ecology Progress Series, 536, 1-9.

[24] SANCHEZ-LORENZO, A., CALBÓ, J. eta WILD, M. 2013. «Global and diffuse solar radiation in Spain: building a homogeneous dataset and assessing their trends». Global and Planetary Change, 100, 343-352.

[25] QUINTANO, E., GANCEDO, U., DÍEZ, I., FIGUEROA, F.L. eta GOROSTIAGA, J.M. 2013. «Solar radiation (PAR and UVA) and water temperature in relation to biochemical performance of Gelidium corneum (Gelidiales, Rhodophyta) in subtidal bottoms off the Basque coast». Journal of Sea Research, 83, 47-55.

[26] LLOPE, M. eta ANADÓN, R. 2007. «In Sea surface warming in the southern Bay of Biscay modulated by oceanic advection» ICES Annual Science Conference. Advice Book 7, 1-24.

[27] VALDÉS, L., LOPEZ-URRUTIA, A., CABAL, J., ALVAREZ-OSSORIO, M., BODE, A. eta MIRANDA, A. 2007. «A decade of sampling in the Bay of Biscay: what are the zooplankton time series telling us? ». Progress in Oceanography, 74, 98-114.

[28] BORJA, A., CHUST, G., FONTÁN, A., GARMENDIA, J.M. eta UYARRA, M.C. 2018. «Long-term decline of the canopy-forming algae Gelidium corneum, associated to extreme wave events and reduced sunlight hours, in the southeastern Bay of Biscay». Estuarine, Coastal and Shelf Science, 205, 152-160.

[29] LLOPE, A., ANADON, R., VIESCA. L., QUEVEDO, M., GONZALEZQUIRÓS, R. eta STENSETH, N.C. 2006. «Hydrography of the southern Bay of Biscay shelf-break region: Integrating the multiscale physical variability over the period 1993-2003». Journal of Geophysical Research: Oceans, 111, $1-14$.

[30] RINNE, H., SALOVIUS-LAUREN, S. eta MATTILA, J. 2011 «The occurrence and depth penetration of macroalgae along environmental gradients in the northern Baltic Sea». Estuarine, Coastal and Shelf Science, 94, 182-191.

[31] STRAIN, E., THOMSON, R.J., MICHELI, F., MANCUSO, F.P. eta AIROLDI, L. 2014. «Identifying the interacting roles of stressors in driving the global loss of canopy-forming to matforming algae in marine ecosystems». Global Change Biology, 20, 3300-3312.

[32] DUFFY, J.E., CARDINALE, B.J., FRANCE, K.E., MCINTYRE, P., THÉBAULT, E. eta LOREAU, M. 2007. «The functional role of biodiversity in ecosystems: incorporating trophic complexity». Ecology Letters, 10 , 522-538. 
[33] NORDERHAUG, K.M. eta CHRISTIE, H. 2011. «Secondary production in a Laminaria hyperborea kelp forest and variation according to wave exposure». Estuarine, Coastal and Shelf Science, 95, 135-144.

[34] GUINDA, X., JUANES, J.A., PUENTE, A. eta ECHAVARRI-ERASUN, B. 2012. «Spatial distribution pattern analysis of subtidal macroalgae assemblages by a non-destructive rapid assessment method». Journal of Sea Research, 67, 34-43.

[35] GOROSTIAGA, J.M. 1994. "Growth and production of the red alga Gelidium sesquipedale of the Basque coast (northern Spain)». Marine Biology, 120, 311-322.

[36] VAN DEN HOEK, C. eta BREEMAN, A.M. 1990. «Seaweed biogeography of the North Atlantic: where are we now?» In: Garbary, D.J., South, G.R. (eds.), Evolutionary Biogeography of the Marine Algae of North Atlantic. Springer-Verlag, Berlin, 55-86.

[37] VALENCIA, V., FRANCO, J., BORJA, A. eta FONTÁN, A. 2004. «Hydrography of the southeastern Bay of Biscay». In: Borja, A., Collins, M. (eds.), Oceanography and Marine Environment of the Basque Country. Elsevier Oceanography Series 70. Elsevier, Amsterdam, 159-194.

[38] DÍEZ, I., MUGUERZA, N., SANTOLARIA, A., GANZEDO, U. eta GOROSTIAGA, J.M. 2012. «Seaweed assemblage changes in the eastern Cantabrian Sea and their potential relationship to climate change». Estuarine Coastal and Shelf Science, 99, 108-120.

[39] CLARKE, K.R. 1993. «Non-parametric multivariate analyses of changes in community structure». Australian Journal of Ecology, 18, 117-143.

[40] SOMERFIELD, P.J. eta CLARKE, K.R. 2013. «Inverse analysis in non-parametric multivariate analyses: distinguishing groups of associated species which covary coherently across samples». Journal of Experimenntal Marine Biology and Ecology, 449, 261-273.

[41] ANDERSON, M.J., GORLEY, R.N. eta CLARKE, K.R. 2008. «PERMANOVA+ for PRIMER: Guide to Software and Statistical Methods» PRIMER-E, Plymouth, Plymouth.

[42] CLARKE, K.R., GORLEY, R.N. 2015. «PRIMER v7: User Manual/tutorial». PRIMER-E, Plymouth.

[43] MINEUR, F., ARENAS, F., ASSIS, J., DAVIES, A.J., ENGELEN, A.H., FERNANDES, F., MALTA, E., THIBAUT, T., VAN NGUYEN T., VAZPINTO, F., VRANKEN, S., SERRÃO, E.A. eta DE CLERCK, O. 2015. «European seaweeds under pressure: Consequences for communities and ecosystem functioning». Journal of Sea Research, 98, 91-108.

[44] HARLEY, C.D.G. eta PAINE, R.T. 2009. «Contingencies and compounded rare perturbations dictate sudden distributional shifts during periods of gradual climate change». Proceedings of the National Academy of Sciences of the United States of America. 106, 11172-11176.

[45] STUART-SMITH, R.D., BARRETT, N.S., STEVENSON, D.G. eta EDGAR, G.J. 2010. «Stability in temperate reef communities over a decadal 
Nahiara Muguerza, María Bustamante, Isabel Díez, Endika Quintano, Javier Tajadura, Iñaki Saiz-Salinas, José María Gorostiaga

time scale despite concurrent ocean warming». Global Change Biology, 16, 122-134.

[46] DUARTE, C.M. eta CEBRIAN, J. 1996. «The fate of marine autotrophic production». Limnology and Oceanography, 41, 1758-1766.

[47] CONNELL, J.H. 1978. «Diversity in tropical rain forests and coral reefs». Science, 199, 1302-1310.

[48] WAHL, M., LiNK, H., ALEXANDRIDIS, N., THOMASON, J.C., CIFUENTES, M., COSTELlO, M.J, DA GAMA, B. A. P., HILLOCK, K. , HOBDAY, A.J., KAUFMANN, M.J., KELLER, S., KRAUFVELIN, P., KRÜGER, I.,LAUTERBACH, L., ANTUNES, B.L., MOLIS, M., NAKAOKA, M., NYSTRÖM, J., BIN RADZI, Z., STOCKHAUSEN, B., THIEL, M., VANCE, T., WESELOH, A., WHITTLE, M., WIESMANN, L.,WUNDERER, L., YAMAKITA, T. eta LENZ, M. 2011. «Re-structuring of marine communities exposed to environmental change: a global study on the interactive effects of species and functional richness». PLOS ONE, 5, e19514.

[49] BREMNER, J., ROGERS, S.I. eta FRID, C.L.J. 2006. «Methods for describing ecological functioning of marine benthic assemblages using biological traits analysis (BTA)». Ecological Indicators, 6, 609-622. 